\title{
Zotepine loaded lipid nanoparticles for oral delivery: development, characterization, and in vivo pharmacokinetic studies
}

\author{
B. Nagaraj, , C. Tirumalesh, S. Dinesh and D. Narendar
}

\begin{abstract}
Background: The purpose of this work was to prepare and evaluate the zotepine (ZT) loaded solid lipid nanoparticles (SLNs) that might improve the oral bioavailability. ZT is an anti-psychotic drug used for the treatment of schizophrenia. Currently, it is available as parenteral and oral dosage form. But, ZT has a poor oral bioavailability of about 7-13\% due to limited aqueous solubility and first-pass effect. ZT-SLNs were developed using homogenization method and characterized for optimal system based on physicochemical characteristics and in vitro release. The optimized ZT-SLNs were evaluated for permeation through rat intestine using evert sac method. The crystalline nature of the ZT-SLNs was studied using DSC and XRD analysis. Surface morphology studies were conducted using SEM. Physical stability of the optimized ZT-SLN was evaluated at refrigerator and room temperature over 2 months. Further, pharmacokinetic (PK) studies of ZT-SLN were conducted in male Wistar rats, in comparison with ZT coarse suspension (ZT-CS), in vivo.

Results: Among all the developed ZT-SLN formulations, optimized formulation (F1) showed Z-avg, PDI, and ZP of 104.3 $\pm 1.6 \mathrm{~nm}, 0.17 \pm 0.01$, and $-30.5 \pm 2.5 \mathrm{mV}$, respectively. In vitro release and permeation studies exhibited $82.9 \pm 1.6 \%$ of drug release and $19.6 \pm 2.1 \%$ of percentage drug permeation over $48 \mathrm{~h}$ and $120 \mathrm{~min}$, respectively. DSC and XRD studies revealed the conversion of ZT to amorphous form. SEM studies showed spherical shape with improved PDI of ZT-SLN formulation. PK studies showed a significant $(p<0.05)$ improvement in AUC of about 1.3-fold, in comparison with ZT-CS in Wistar rats.
\end{abstract}

Conclusion: Therefore, the results concluded that SLNS could be considered as a new alternative delivery system for the enhancement of oral bioavailability of ZT.

Keywords: Zotepine, Solid lipid nanoparticles, Crystallinity, In vitro release, Ex vivo permeation, Oral bioavailability

\section{Background}

The amount of the drug reaching to the systemic circulation mainly depends on the rate of absorption, which in turn depends on the drug solubility [1]. Oral delivery of the drugs is the most conventional and widely used technique for the administration of majority of active moieties. However, several compounds are unsuccessful and failed owing to their low absorption and low bioavailability upon oral administration [2]. The drugs with poor oral

\footnotetext{
* Correspondence: bnrpharmacy@kakatiya.ac.in; dnrku14@gmail.com Nanotechnology and Novel Drug Delivery Laboratory, Department of Pharmaceutics, University College of Pharmaceutical Sciences, Kakatiya University, Warangal Telangana-506009, India
}

bioavailability are unable to reach the minimum effective concentration to exhibit therapeutic action. Some of the reasons for poor bioavailability include poor solubility, inappropriate partition coefficient as it influences the permeation of drug through lipid membrane, first-pass metabolism, P-glycoprotein (P-gp) mediated efflux, and degradation of drug in the gastrointestinal tract (GIT) due to $\mathrm{pH}$ of the stomach or enzymatic degradation or by chemical [3].

To overcome the solubility and oral bioavailability problems of biopharmaceutical classification system (BCS) classes II and IV drugs, the research in the present scenario is focused on bioavailability improvement, by

\section{Springer Open}

(c) The Author(s). 2020 Open Access This article is licensed under a Creative Commons Attribution 4.0 International License, which permits use, sharing, adaptation, distribution and reproduction in any medium or format, as long as you give appropriate credit to the original author(s) and the source, provide a link to the Creative Commons licence, and indicate if changes were made. The images or other third party material in this article are included in the article's Creative Commons licence, unless indicated otherwise in a credit line to the material. If material is not included in the article's Creative Commons licence and your intended use is not permitted by statutory regulation or exceeds the permitted use, you will need to obtain permission directly from the copyright holder. To view a copy of this licence, visit http://creativecommons.org/licenses/by/4.0/. 
using various solubility enhancement techniques like liquisolid compacts $[4,5]$, micronization using nanosuspensions [6], solid dispersions using complexation [7, 8], and salt formation [9]. Majority of the drugs undergo first-pass effect after oral administration; thereby, the therapeutic concentration required in the systemic circulation is lowered. However, by using the lipid-based delivery systems, both the solubility and first-pass effect problems are resolved, and subsequently oral absorption was improved [3].

Lipid-based drug delivery systems are one of the approaches, which favors the lymphatic transport of oral administered drugs by escaping the first-pass effect $[10$, 11]. Colloidal lipid nanocarriers such as solid lipid nanoparticles (SLNs) and nanostructured lipid carriers (NLCs) are the promising approaches in the lipid-based delivery systems. SLNs are mainly developed with the solid lipid carrier, used for incorporation of drug, and lipid dispersion is stabilized by the use of biocompatible surfactants [12]. SLNs offer remarkable advantages such as low toxicity, high bioavailability (pharmacokinetic and pharmacodynamic) of drugs, versatility of incorporation of both hydrophilic and lipophilic drugs, and feasibility of large-scale production. SLN formulations have controlled drug release properties and provide enhanced chemical stability of drug molecules [13]. SLNs also used for drug targeting to various tissues such as the liver [14] and ocular [15] for treatment of various diseases.

Zotepine (ZT) is an atypical anti-psychotic drug, which belongs to BCS class II category. The mechanism of action of ZT is mediated through combined dopamine and serotonin antagonisms [16]. It has a poor oral bioavailability of about $7-13 \%$, due to poor aqueous solubility $(0.046 \mu \mathrm{g} / \mathrm{L})$, highly lipophilic $(\log p 5.6)$, and also hepatic first-pass metabolism. The drug levels were also decreased in the presence of CYP enzyme $[17,18]$. In oral route, $30 \%$ of the drug metabolizes into nor-zotepine (active metabolite), and remaining $70 \%$ of drug transforms to inactive metabolites such as 3-hydroxyzotepine, 2-hydroxyzotepine, and zotepine-S-oxide [16]. Previously, Pailla et al. [19] developed intranasal delivery of ZT for enhanced brain targeting, using nanosuspension formulation. The enhanced solubility and in vitro dissolution rate of ZT was observed and reported with selfemulsifying drug delivery systems [20]. But, till now there are no lipid nanocarrier systems were reported for enhanced oral delivery of ZT. Hence, in this attempt, ZT loaded SLNs were developed that might improve the oral bioavailability.

The objective of the present investigation was aimed to develop and characterize ZT loaded SLNs for enhanced oral delivery. Accordingly, ZT-SLNs were prepared using film hydration method and evaluated for an optimized formulation based on the physical and chemical parameters, in vitro release studies. Further, optimized formulation also characterized for solid state characterization for crystallinity, SEM for surface morphology, and ex vivo permeation studies. In vivo performance of optimized ZT-SLN was observed by pharmacokinetic (PK) studies in male Wistar rats, comparison with ZT-coarse suspension (ZT-CS) as control formulation.

\section{Methods}

Zotepine was a kind gifted sample from Symed labs, Hyderabad, India. Dynasan ${ }^{\circ} 118$ and Dynasan ${ }^{\circ} 114$ were purchased from Sigma-Aldrich, Hyderabad, India. Compritol $^{\circ} 888$ ATO was gift sample from Neuheit Pharma Technologies Private Ltd., Hyderabad. Soylecithin was a gifted sample from Lipoid, Ludwigshafen, Germany. Captex 355 and Captex 200 were gift samples from Abitec Corporation. All other chemicals were of analytical grade, and solvents were of HPLC grade.

\section{Animals}

Male Wister rats $(210 \pm 30 \mathrm{~g})$ used for the study were procured from Teena Biolabs Pvt Ltd., Hyderabad, India. The studies were conducted with prior approval of Institutional Animal Ethical Committee, Kakatiya University, India (IAEC/01/UCPSC/KU/2018).

\section{Solubility studies}

ZT solubility in various solid lipids was determined by using shaking method. Solid lipids were heated to above $5{ }^{\circ} \mathrm{C}$ of their melting points. An excess amount of ZT was added and was continuously stirred on gyrator shaker at $180 \mathrm{rpm}$ for $48 \mathrm{~h}$. The supernatant was collected and filtered through $0.45-\mu$ membrane filter after centrifugation [21]. Necessary dilutions were made to the filtrate with methanol and estimated the drug concentration using UV-Visible spectrophotometer at $\lambda_{\max }$ of $261 \mathrm{~nm}$ (SL-159, Elico, Hyderabad, India). Simultaneously, solubility of ZT in release media such as distilled water, $0.1 \mathrm{~N} \mathrm{HCl}(\mathrm{pH} 1.2)$, phosphate buffer of $\mathrm{pH} 6.8$ and $\mathrm{p} \mathrm{H}$ 7.4.

\section{Preparation of zotepine loaded solid lipid nanoparticles} Zotepine loaded solid lipid nanoparticles (ZT-SLNs) were prepared using homogenization-probe sonication method, based on film hydration method [22]. Required amounts of ZT, solid lipid, and soy lecithin were dissolved in $10 \mathrm{~mL}$ mixture of chloroform and methanol $(1: 1)$ in a round bottom flask. Organic solvents were removed using rota evaporator (Heidolph, Germany). Drug embedded lipid layer was molten by heating at $5{ }^{\circ} \mathrm{C}$ above the melting point of the solid lipid. Aqueous phase was prepared by dissolving Poloxamer-188 in double distilled water and heated to the same 
temperature of oil phase. Hot aqueous phase was added to oil phase. Homogenization was carried out at 12,000 $\mathrm{rpm}$ for $5 \mathrm{~min}$ to form pre-emulsion. The obtained emulsion was ultra-sonicated using Probe soniactor with $12 \mathrm{~T}$ probe tip (Bandelin, Germany) for $20 \mathrm{~min}$. ZTSLNs were obtained upon cooling to room temperature. The compositions of the SLNs are given in Table 1.

\section{Preparation of ZT coarse suspension (ZT-CS)}

About $100 \mathrm{mg}$ of sodium carboxy methyl cellulose (suspending agent) was taken in a mortar and triturated. Then, $10 \mathrm{mg}$ of ZT was added to it and together triturated. To it, $10 \mathrm{~mL}$ of double distilled water was added and again triturated for about $5 \mathrm{~min}$ to obtain ZT-CS (1 $\mathrm{mg} / \mathrm{mL}$ ). This was used as control formulation.

\section{Characterization of ZT-SLNs}

Measurement of particle size, polydispersity index (PDI), and zeta potential (ZP)

The mean sizes as $\mathrm{Z}$-avg $(\mathrm{nm})$, polydispersity index (PDI), and zeta potential (ZP) of ZT-SLNs were measured by photon correlation spectroscopy (PCS) using a Malvern Zetasizer (Nano ZS90, UK). About $100 \mu \mathrm{L}$ of prepared SLN formulation was diluted to $5 \mathrm{~mL}$ with double distilled water to get optimum kilo counts per second (Kcps) of 50-200 for measurements [23]. All measurements were in triplicate at $25^{\circ} \mathrm{C}$.

\section{Drug Content and entrapment efficiency (EE)}

About $0.1 \mathrm{~mL}$ of ZT-SLN formulation was taken and diluted with chloroform to methanol (1:1), and drug content of the diluted samples was estimated by HPLC method. The entrapment efficiency (EE, \%) in ZT-SLNs was calculated by estimating the concentration of the free drug in the aqueous phase of an undiluted formulation, using an ultrafiltration method centrisort tubes (Sartorius, Germany). The aqueous medium was separated by ultra-filtration using centrisort tubes, which consisting a filter membrane (M.Wt.20,000 Da) at the base of the sample recovery chamber. Centrifugation was carried at $4000 \mathrm{rpm}$ for $30 \mathrm{~min}$ [24]. The SLN along with the encapsulated drug remained in outer chamber, and aqueous phase moved into sample recovery chamber through filter membrane. The amount of zotepine in aqueous phase was estimated by HPLC method. The EE was calculated by using the Eq. 1:

$$
0 /{ }_{0} \mathrm{EE}=\left[\frac{D \mathrm{i}-D \mathrm{f}}{D \mathrm{i}}\right] \times 100
$$

where $D_{\mathrm{i}}$ is the total drug content and $D_{\mathrm{f}}$ is the free drug present in the aqueous phase.

\section{In vitro drug release studies}

The in vitro drug release study was performed using dialysis method. Dialysis membrane (Hi-Media, Hyderabad, India) having average pore size $2.4 \mathrm{~nm}$ and molecular weight cut-off between12,000-14,000 Da were used for the release studies [23, 25]. Dialysis membrane was soaked overnight in double distilled water prior to the study. Release studies were carried out for first $2 \mathrm{~h}$ in $0.1 \mathrm{~N} \mathrm{HCl}$, followed by $\mathrm{pH} 6.8$ phosphate buffer for $46 \mathrm{~h}$ by open tube method. Dialysis membrane was tied to open tube which acts as a donor compartment. Temperature of receptor compartment was maintained at $37 \pm 0.5{ }^{\circ} \mathrm{C}$. Aliquot of $2 \mathrm{~mL}$ sample was withdrawn at regular time points of $0,0.25,0.5,1,2,3,4,6,8,10,12$, 24,36 , and $48 \mathrm{~h}$ from receiver compartment and replenished with same volume of fresh buffer. The collected samples were suitably diluted and analyzed by UVVisible spectrophotometer.

\section{Ex vivo studies by normal sac method}

Ex vivo studies were performed for optimized ZT-SLN formulation by using evert sac method [26]. The studies were conducted with prior approval of Institutional Animal Ethical Committee (IAEC/01/UCPSC /KU/2018). Evert sac studies were carried out in order to investigate the permeation behavior of the drug solution and optimized formulation $(1 \mathrm{~mL})$ across the small intestine. In this study, male Wistar rats of $210 \pm 30 \mathrm{~g}(n=3)$ were taken and subjected to overnight fasting. The rats were

Table 1 Composition of zotepine loaded solid lipid nanoparticles

\begin{tabular}{|c|c|c|c|c|c|c|c|}
\hline Ingredients (\% w/v) & F1 & $\mathrm{F} 2$ & F3 & F4 & F5 & F6 & F7 \\
\hline Zotepine (mg) & 0.1 & 0.1 & 0.1 & 0.1 & 0.1 & 0.1 & 0.1 \\
\hline Dynasan ${ }^{\oplus} 118$ & 1.0 & - & - & 2.0 & - & - & - \\
\hline Dynasan ${ }^{\circledast 114}$ & - & 1.0 & - & - & 2.0 & - & - \\
\hline Compritol ATO 888 & - & - & 1.0 & - & - & 2.0 & - \\
\hline Soylecithin & 1.0 & 1.0 & 1.0 & 1.0 & 1.0 & 1.0 & - \\
\hline Poloxamer 188 & 1.5 & 1.5 & 1.5 & 1.5 & 1.5 & 1.5 & - \\
\hline $\mathrm{Na} \mathrm{CMC} \mathrm{C}^{*}$ & - & - & - & - & - & - & 0.05 \\
\hline Water $(\mathrm{mL})$ & QS 10 & QS 10 & QS 10 & QS 10 & QS 10 & QS 10 & QS 10 \\
\hline
\end{tabular}

"Na CMC, sodium carboxy methyl cellulose; F7, coarse suspension of ZT 
sacrificed by cervical dislocation technique, and jejunum of $4 \mathrm{~cm}^{2}$ were isolated, flushed with saline solution, and transferred into oxygenated Krebs's ringer solution. The one end of the sac was tied with thread and filled with the optimized ZT-SLN or ZT-CS as control $(1 \mathrm{~mL})$ formulation, and the other end was tied. The segment was immersed in $100 \mathrm{~mL}$ in Krebs's ringer solution, and the medium was oxygenated using aerator. At regular time intervals $(0,15,30,45,60,90$, and $120 \mathrm{~min})$, samples were withdrawn from beaker and analyzed for drug content [27].

\section{Lyophilization of ZT-SLNs}

The optimized ZT-SLN formulation was subjected to lyophilization. The SLN formulation was prepared, using $10 \% \mathrm{w} / \mathrm{w}$ of trehalose as cryoprotectant. Prepared SLN formulation was kept in $-80{ }^{\circ} \mathrm{C}$ freezer for overnight and subjected to lyophilization (Lyodel, Chennai, India) with applied vacuum [28]. Lyophilized formulation was subjected to particle size analysis, drug content, EE, and in vitro release before and after lyophilization.

\section{Solid-state characterization}

\section{Differential scanning calorimetry}

Differential scanning calorimetry (DSC) of sample was performed through using Perkin Elmer DSC 4000 model to find out the presence of any interaction between drug and the excipients and also to check the alterations in crystallinity of the drug. About $8 \mathrm{mg}$ of the pure drug, physical mixture and optimized lyophilized ZT-SLN formulation samples were taken in aluminum pans, using dry nitrogen as purging gas. The heating rate was $10^{\circ} \mathrm{C} /$ $\mathrm{min}$, and the obtained thermograms were observed for any type of interaction [29]. Empty pan was used as reference for the study.

\section{$X$-ray diffraction studies}

Crystalline nature of the nanoparticle formulation was analyzed through powder X-ray diffractometer (XRD6000, Shimazdu, Japan) [25]. Powder XRD studies were performed on the samples by exposing them to nickel filtered $\mathrm{CuK} \alpha$ radiation $(40 \mathrm{kV}, 30 \mathrm{~mA})$ and scanned from 2 to $70^{\circ}, 2 \theta$ at a step size of $0.045^{\circ}$ and step time of
$0.5 \mathrm{~s}$. Samples used for PXRD analysis were pure ZT, pure lipid, and lyophilized ZT loaded solid lipid nanoparticles [30].

Surface morphology by scanning electron microscopy (SEM) The morphology of pure ZT and freeze-dried ZT-SLN formulation was investigated by scanning electron microscope (SEM S-3700, Hitachi, Japan). The samples were fixed on a brass stub and were coated by thin layer of gold to make electrically conductive [25]. SEM images were recorded at applied fixed voltage.

\section{Bioavailability study}

\section{Study design and sampling schedule}

Healthy male Wistar rats (weighing $210 \pm 30$ g) were used for the PK study. The animals were fasted overnight and had free access to water. The studies were conducted with prior approval of Institutional Animal Ethical Committee (IAEC/01/UCPSC /KU/2018). The animals were divided into two groups $(n=6)$ and were orally administered with ZT-CS (F7) and optimized ZTSLN (F1) formulation at a dose of $2.2 \mathrm{mg} / \mathrm{kg}$ body weight with the help of rat oral feeding tube. At appropriate predetermined time intervals after oral administration, blood samples were collected $(0,0.5,1,2,4,6,8,10,12$, $24,48,72$, and $96 \mathrm{~h}$ ) by retro-orbital venous plexus puncture. The blood samples were allowed to clot and centrifuged for $15 \mathrm{~min}$ at $3000 \mathrm{rpm}$. The serum was separated and transferred into clean micro centrifuge tubes and stored at $-20{ }^{\circ} \mathrm{C}$ until analysis.

\section{HPLC method}

HPLC analysis was conducted with $\mathrm{C}_{18}$ column $(5 \mu \mathrm{m}$; $250 \mathrm{~mm} \times 4.6 \mathrm{~mm})$. Mobile phase consisting of 45:55 (v/ v) ratio of acetonitrile and phosphate buffer adjusted to $\mathrm{pH} 4.7$ at a flow rate of $1 \mathrm{~mL} / \mathrm{min}$. Wavelength of 261 $\mathrm{nm}$ was used for detection of peaks. The retention times of ZT and ramipril (used as internal standard; IS) were found to be $7.8 \mathrm{~min}$ and $4.5 \mathrm{~min}$, respectively.

\section{Extraction procedure from rat serum sample}

To $100 \mu \mathrm{L}$ of serum, $100 \mu \mathrm{L}$ of IS (ramipril, $2 \mu \mathrm{g} / \mathrm{mL}$ ), and $300 \mu \mathrm{L}$ of methanol were added. After vortex mixing

Table 2 Physicochemical characteristics - size, PDI, zeta potential, entrapment efficiency, and assay of zotepine loaded solid lipid nanoparticle formulations (mean $\pm \mathrm{SD}, n=3$ )

\begin{tabular}{llllll}
\hline Formulation & Size $(\mathrm{nm})$ & PDI & ZP $(\mathrm{mV})$ & Assay $(\mathrm{mg})$ & EE $(\%)$ \\
\hline F1 & $104.3 \pm 2.4$ & $0.17 \pm 0.01$ & $-30.5 \pm 2.5$ & $9.8 \pm 0.3$ & $98.4 \pm 2.8$ \\
F2 & $121.7 \pm 3.1$ & $0.21 \pm 0.06$ & $-29.8 \pm 1.4$ & $9.5 \pm 0.4$ & $97.3 \pm 1.6$ \\
F3 & $147.3 \pm 2.8$ & $0.61 \pm 0.05$ & $-10.6 \pm 0.6$ & $9.8 \pm 0.6$ & $96.1 \pm 3.2$ \\
F4 & $259.2 \pm 1.6$ & $0.34 \pm 0.03$ & $-28.4 \pm 1.7$ & $9.6 \pm 0.5$ & $91.1 \pm 1.5$ \\
F5 & $340.4 \pm 3.0$ & $0.29 \pm 0.07$ & $-29.4 \pm 2.1$ & $8.9 \pm 0.1$ & $93.1 \pm 1.8$ \\
F6 & $303.3 \pm 2.1$ & $0.50 \pm 0.08$ & $-16.6 \pm 1.0$ & $8.7 \pm 0.4$ & $91.4 \pm 2.1$ \\
\hline
\end{tabular}




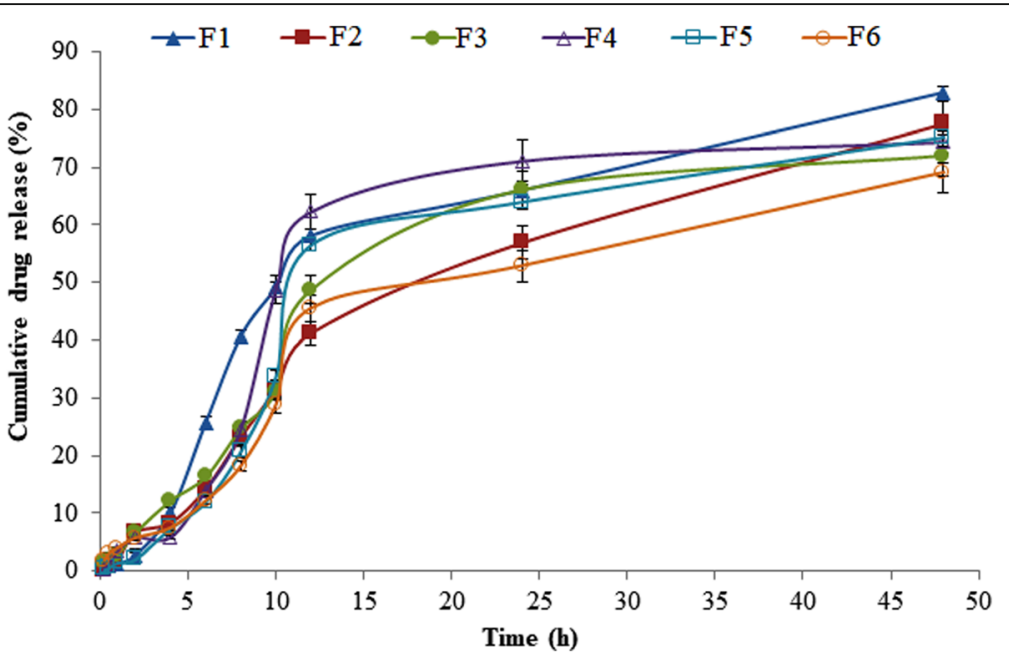

Fig. 1 In vitro release profiles of zotepine from ZT-SLNs (mean $\pm S D, n=6$ )

for $5 \mathrm{~min}$ at room temperature, the samples were centrifuged at $5000 \mathrm{rpm}$ for $15 \mathrm{~min}$. After centrifugation, the whole organic layer was separated and transferred into another eppendorf tube. Finally, a volume of $20 \mu \mathrm{L}$ was injected into HPLC system.

\section{Estimation of pharmacokinetic parameters and statistical significance}

The Kinetica 2000 software (version 5.0, USA) was used for the estimation of PK parameters like $C_{\max }, T_{\max }$, $\mathrm{AUC}_{\text {total }}, t_{1 / 2}$, and MRT, and the values were expressed in mean $\pm \mathrm{SD}$. One-way ANOVA was performed using the Graph pad prism software (version 5.03) for statistical comparison of data at $p$ value of 0.05 .

\section{Physical stability studies}

An optimized ZT-SLN formulation was stored at room temperature and refrigerated temperature for 2 months. Z-avg, PDI, ZP, assay, and EE of the samples were observed at predetermined time intervals.

\section{Results}

\section{Solubility studies}

Solubility of ZT was studied in various solid lipids. Dynasan $^{\circledR} 118$, Dynasan ${ }^{\circledR} 114$, and Compritol $^{\odot}$ ATO 888 were selected as solid lipids for SLN formulations development. Order of solubility of ZT in solid lipids was Dynasan $^{\oplus} 118>$ Dynasan $^{\oplus} 114>$ Compritol $^{\oplus}$ ATO888> $^{\circ}$ Dynasan $^{\oplus} 112>$ Precirol $^{\oplus}$ ATO5. The solubility of ZT was decreased with increased $\mathrm{pH}$ of dissolution media and

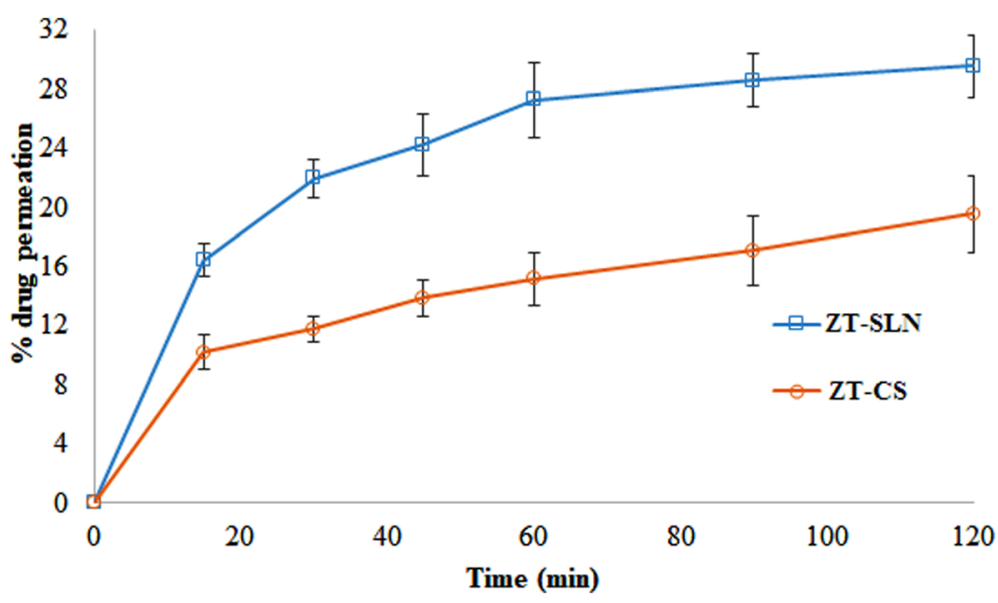

Fig. 2 Ex vivo permeation profiles of zotepine from ZT-CS (F7) and ZT-SLN (F1) formulations (mean \pm SD, $n=3$ ) 
Table 3 Physicochemical characteristics of optimized ZT-SLN before and after lyophilization (mean \pm SD, $n=3$ )

\begin{tabular}{llllll}
\hline Condition & Size $(\mathrm{nm})$ & PDI & ZP $(\mathrm{mV})$ & Assay (mg) & EE (\%) \\
\hline Pre-lyo & $109.4 \pm 1.7$ & $0.18 \pm 0.03$ & $-30.1 \pm 1.9$ & $9.9 \pm 0.2$ & $98.0 \pm 2.1$ \\
Post-lyo & $272.8 \pm 4.1$ & $0.42 \pm 0.09$ & $-29.5 \pm 2.1$ & $9.8 \pm 0.1$ & $97.9 \pm 2.8$
\end{tabular}

indicates $\mathrm{pH}$ dependent solubility. The order of solubility in release media was as follows: $0.1 \mathrm{~N} \mathrm{HCl}>\mathrm{pH} 6.8$ phosphate buffer $\gg$ water $>\mathrm{pH} 7.4$ phosphate buffer.

\section{Characterization of ZT-SLNs}

\section{Measurement of particle size, PDI, and ZP}

Prepared ZT-SLNs were characterized for particle size, PDI, and ZP, using Zetasizer and reported in Table 2. Particle size, PDI, and ZP of the ZT-SLNs formulations (F1-F6) were $104.3 \pm 2.4$ to $340.4 \pm 3.0 \mathrm{~nm}, 0.17 \pm 0.01$ to $0.61 \pm 0.05$, and $-10.66 \pm 0.6$ to $-30.5 \pm 2.5 \mathrm{mV}$.

\section{Drug content and entrapment efficiency}

All the formulations were analyzed for EE and total drug content by HPLC (Table 2). EE of the all the formulations was ranged from $91.1 \pm 1.5$ to $98.4 \pm 2.8 \%$. Among all the developed formulations, F1 formulation is having the high EE. Drug contents of all the formulations were in the range of $8.7 \pm 0.4$ to $9.8 \pm 0.3 \mathrm{mg}$.

\section{In vitro release studies}

All the prepared SLNs were subjected to in vitro release studies, using dialysis method. In this study, $0.1 \mathrm{~N} \mathrm{HCl}$ pH 1.2 followed by pH 6.8 phosphate buffer for the release studies. In vitro release of ZT from SLN formulations was observed to $69 \pm 2.1$ to $82.9 \pm 3.6 \%$ range, over $48 \mathrm{~h}$ (Fig. 1). However, formulations prepared with $1 \%$ and $2 \% \mathrm{w} / \mathrm{v}$ lipid concentrations were not significantly influence the drug release behavior from the SLN formulations. Formulations prepared with Dynasan ${ }^{\circ} 118$, Dynasan $^{\circ} 114$, and Compritol ATO888 showed $82.9 \pm 3.6$ and $74.4 \pm 2.1 \%$ (from F1 and F2), $77.5 \pm 1.1$ and $75.1 \pm$ $2.9 \%$ (from F3 and F4), and $72 \pm 1.9$ and $69 \pm 2.1 \%$ (from F5 and F6), respectively. The drug release from the ZT-CS (F7) formulation showed $51.9 \pm 2.7 \%$ in 120 min (data not showed). In all the prepared formulations, ZT-SLN of F1 formulation prepared with Dynasan ${ }^{\circ} 118$ was having less particle size and PDI, stable ZP, with high EE, acceptable drug content and also showed maximum drug release of $82.9 \pm 3.6 \%$ in $48 \mathrm{~h}$, comparison with other formulations. Therefore, F1 formulation was

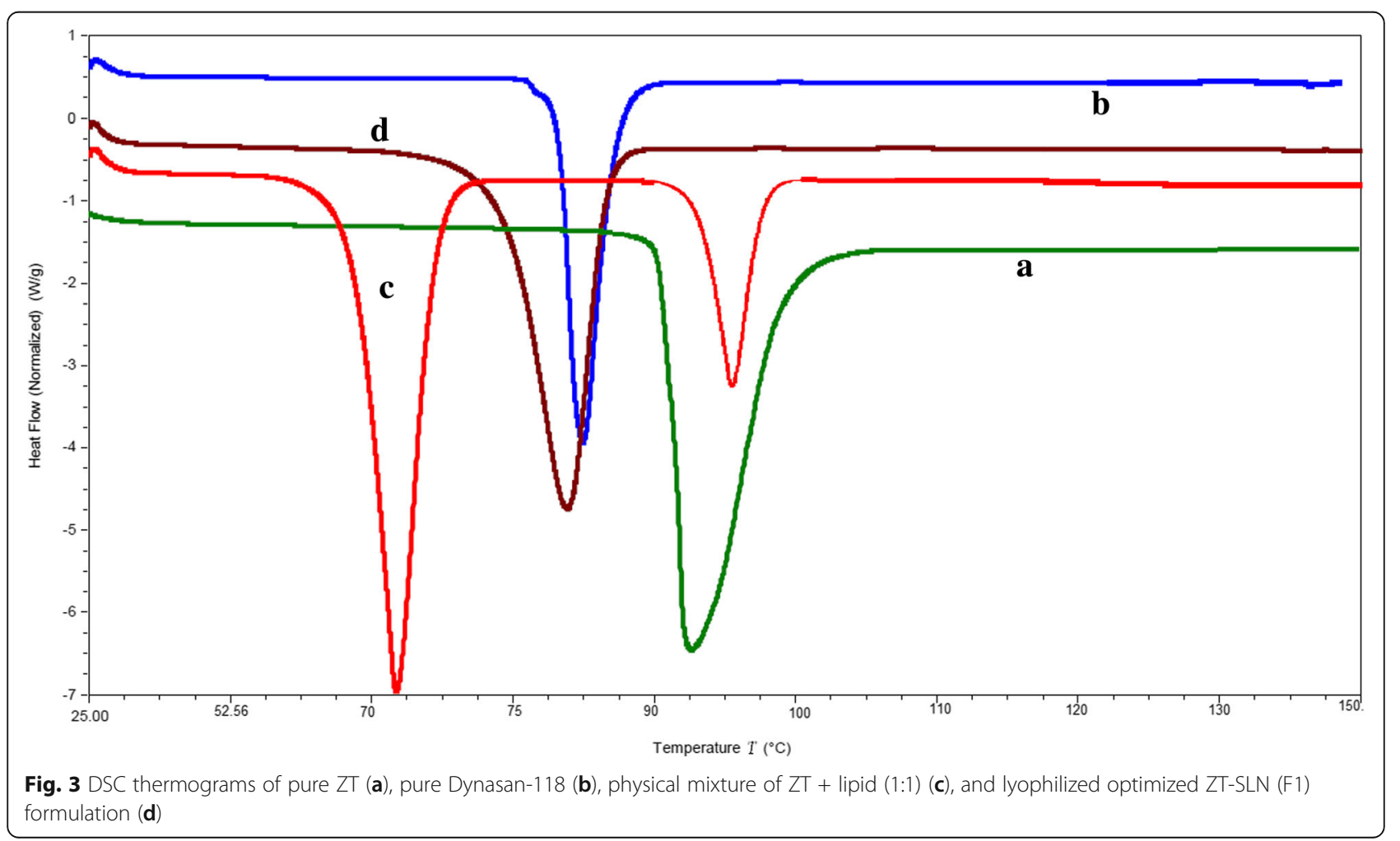


considered as an optimized formulation and selected for further studies.

\section{Ex vivo permeation studies}

Ex vivo permeation studies were performed for optimized ZT-SLN formulation (F1) in comparison with ZT-CS (F7) through rat intestine using everted sac perfusion method. The duration of the study was $120 \mathrm{~min}$ and represented in Fig. 2. From the results, the percentage of ZT permeation from both F7 and F1 formulations was $19.6 \pm 2.1 \%$ and $29.6 \pm 2.6 \%$, respectively. Statistically significance difference $(p<0.05)$ was observed from the F1 and F7 formulations.

\section{Lyophilization of ZT-SLN}

Optimized ZT-SLN formulation was subject to lyophilization, using $10 \% \mathrm{w} / \mathrm{w}$ of trehalose as cryoprotectant. The cryoprotectant and its concentration were selected based on the previously reported methods [24]. The lyophilized formulation was characterized for before and after lyophilization and presented in Table 3.

\section{Solid state characterization}

\section{DSC}

DSC studies were used to determine the compatibility status of the solid lipids and other excipients used in the SLN formulation, crystalline nature of the drug in the nanoformulations and was based on the fact that different lipids possessed different melting points and enthalpies. DSC thermograms of pure ZT, pure Dynasan ${ }^{\circ} 118$, physical mixture of ZT, and Dynasan ${ }^{\circ} 118$ in 1:1 ratio and lyophilized ZT-SLN formulation are shown in Fig. 3. The DSC thermogram of pure ZT showed a sharp endothermic peak at $93.76{ }^{\circ} \mathrm{C}$ (Fig. 3a), and it is corresponding to reported melting point. Pure Dynasan ${ }^{\circ} 118$ showed a sharp endothermic peak at $76.06^{\circ} \mathrm{C}$ (Fig. 3b). Physical mixture of ZT with Dynasan ${ }^{\circ} 118$ showed drug peaks at $94.82^{\circ} \mathrm{C}$, however with less enthalpy compared with pure ZT enthalpy. But, melting endotherm of drug was well preserved with slight changes in terms of shifting in the temperature of the melt (Fig. 3c). The absence of endotherm peak of drug in lyophilized ZT-SLN formulation (Fig. 3d) was observed.

\section{$X R D$}

X-ray diffractometer (XRD) studies of pure ZT, pure lipid, and lyophilized ZT-SLN (F1) were showed in Fig. 4. From the results, powder XRD patterns of ZT showed sharp peaks at $2 \theta$ scattered angles of $10.3,11.4,13.5,17.1,18.3$, $19.8,20.3,23.2$, and $24.1^{\circ}$; these were indicating the crystalline nature of drug. These characteristic peaks of ZT peaks were absent in the lyophilized ZT-SLN sample. This specified that the ZT was not in crystalline form and

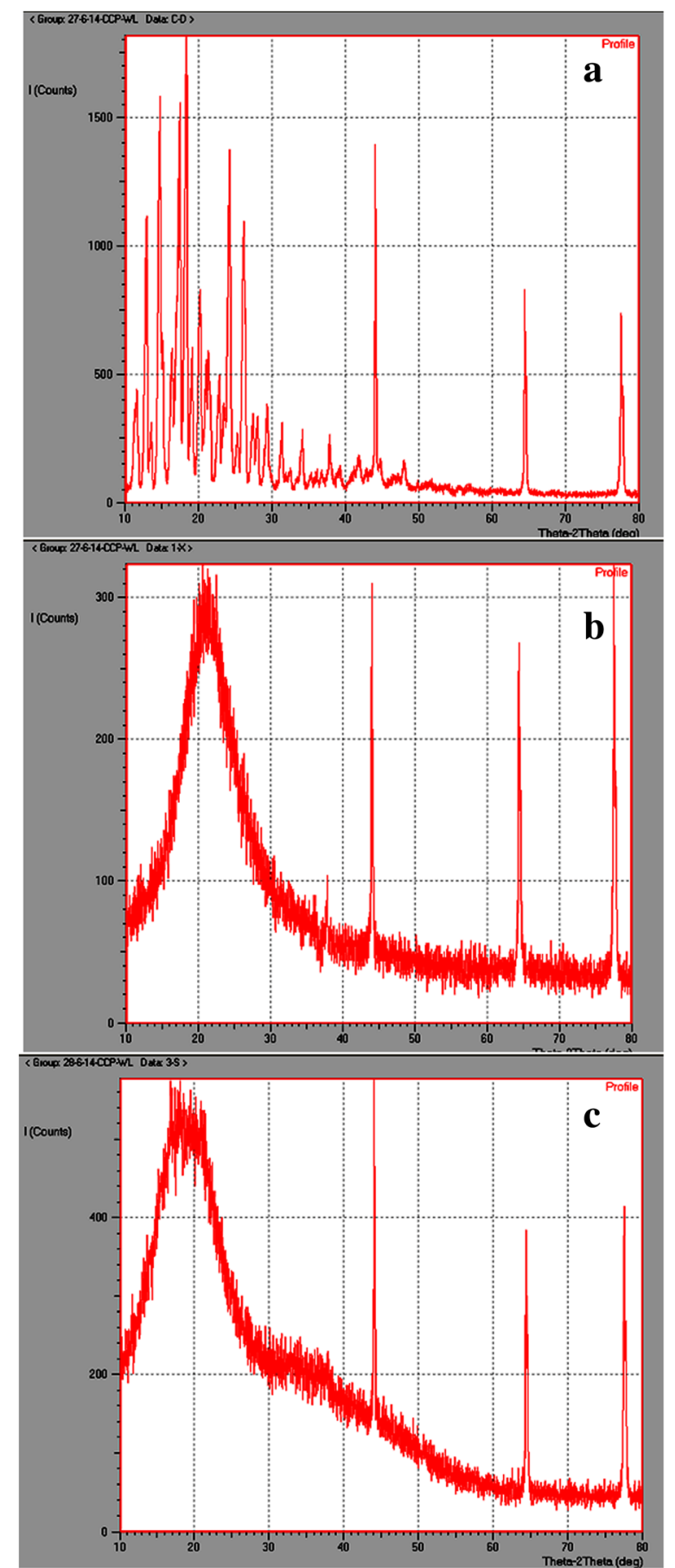

Fig. 4 XRD spectra of pure ZT (a), pure Dynasan-118 (b), and lyophilized optimized ZT-SLN (F1) formulation (c)

converted to amorphous nature, after lyophilization of optimized ZT-SLN (F1).

\section{SEM}

The shape of the ZT before and after loading onto SLN formulation was observed using SEM method. The 


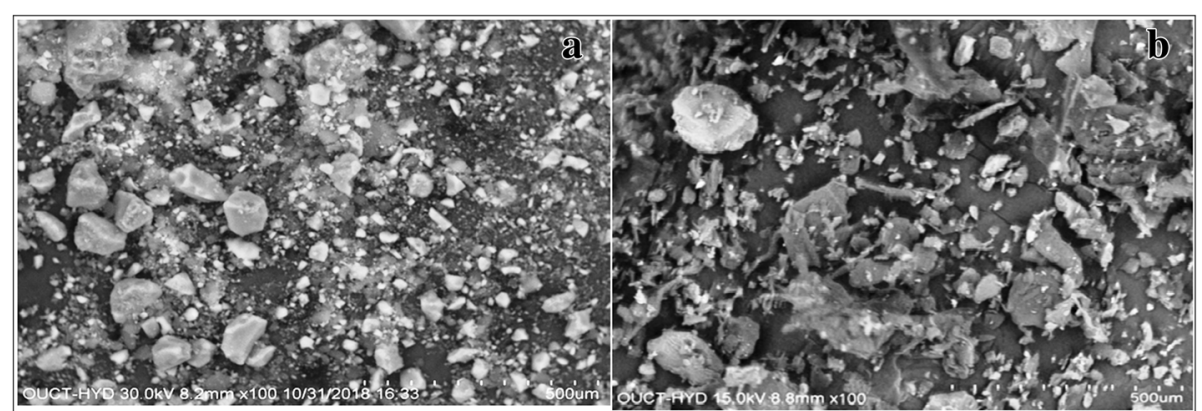

Fig. 5 SEM images of pure ZT (a) and optimized lyophilized ZT-SLN formulation (b)

results are showed in Fig. 5. The results of the SEM studies also correlated with particle size of after reconstituted lyophilized sample.

\section{Stability study of optimized ZT-SLN}

The stability of the optimized ZT-SLN was monitored by storage at room temperature $\left(25^{\circ} \mathrm{C}\right)$ and refrigerated temp $\left(4{ }^{\circ} \mathrm{C}\right)$ for 60 days. The formulations were analyzed on 1st day, 30th day, and 60th day, respectively, for size, PDI, ZP, EE, and drug content. The results indicated that no significant variations were noticed in all the measured parameters and found to be stable up to 2 months (Table 4).

\section{Pharmacokinetic (PK) study}

Oral bioavailability study for ZT-CS (F7) and optimized ZT-SLN (F1) formulations were performed in male Wistar rats. The collected serum samples were analyzed for drug content, using HPLC method. Ramipril is used as IS. Retention times of both ZT and IS were 7.8 and 4.5 min, respectively. The linearity of the $\mathrm{ZT}$ was observed in the concentration range of $0.25-12 \mu \mathrm{g} / \mathrm{mL}$. The limit of detection and limit of quantification of the $\mathrm{ZT}$ were 0.1 and $0.2 \mu \mathrm{g} / \mathrm{mL}$, respectively. About $98.5 \%$ of extraction efficiency was observed from recovery studies.

The PK parameters were calculated using the Kinetica 2000 software. Mean serum concentration vs time profiles of F7 and F1 formulation after oral administration of single dose are shown in Fig. 6. PK parameters like AUC $C_{\text {total }}, C_{\max }, T_{\max }$, MRT, and $t_{1 / 2}$ of the formulations were calculated and shown in Table 5 . A significant difference observed in $C_{\max }$ and MRT of the F1 formulation indicates the prolonged release of the ZT. AUC is the major determining factor of the bioavailability of the formulations. From the results, $\mathrm{AUC}_{\text {total }}$ values of F7 and F1 were found to be $32.3 \pm 1.1$ and $43.3 \pm$ $2.2 \mu \mathrm{g} / \mathrm{mL} . \mathrm{h}$, respectively, and were statistically significant $(p<0.05)$. From the results, about 1.3 -fold improvement in the bioavailability observed from F1 formulation in comparison with F7 formulation.

\section{Discussion}

ZT-SLNs formulations were prepared using three different lipids such as Dynasan ${ }^{\circ} 118$, Dynasan 114 , and Compritol -ATO 888, each at 1 and $2 \%$ w/v, respectively. Homogenization followed by probe sonication method used for the preparation of ZT-SLNs. The homogenization time and probe sonication time used at 12,000 rpm for $5 \mathrm{~min}$ and $20 \mathrm{~min}$ at $40 \%$ amplitude, respectively. The conditions were optimized based on the earlier reported methods.

From the results, as the concentration of lipids increased from $1 \% \mathrm{w} / \mathrm{v}$ to $2 \% \mathrm{w} / \mathrm{v}$, increased in particle size and PDI, decreased in ZP were observed for F1-F4 formulations. But, F5 and F6 formulations showed revere trend. This could be due to the nature of lipid, i.e., Compritol ${ }^{\circ} \mathrm{ATO} 888$. This might be due to the changes in the orientation of the drug and presence of the void spaces of lipid and drug. PDI of the formulations (F1F6) was ranged from 0.17 to 0.61 . The PDI value of up to 0.3 was considered to be homogeneous dispersion and was evidenced from the earlier reports [15]. The formulation ZP of the ZT-SLNs was crucial factor the stability assessment. In this case, the combination of surfactants imparts the electrostatic repulsion and steric

Table 4 Stability studies of optimized formulation of ZT-SLN (F1) at both room temperature $\left(25^{\circ} \mathrm{C}\right)$ and refrigerated $\left(4^{\circ} \mathrm{C}\right)$ for a period 2 months (mean $\pm \mathrm{SD}, n=3$ )

\begin{tabular}{|c|c|c|c|c|c|c|c|c|c|c|}
\hline \multirow{2}{*}{$\begin{array}{l}\text { Time } \\
\text { (day) }\end{array}$} & \multicolumn{5}{|c|}{ At room temperature $\left(25^{\circ} \mathrm{C}\right)$} & \multicolumn{5}{|c|}{ At refrigerated temperature $\left(4^{\circ} \mathrm{C}\right)$} \\
\hline & Size $(n m)$ & PDI & ZP & $\mathrm{EE}$ & Assay & Size $(n m)$ & PDI & Potential & $\mathrm{EE}$ & Assay \\
\hline 1 & $104.3 \pm 2.4$ & $0.17 \pm 0.01$ & $-30.5 \pm 2.5$ & $9.8 \pm 0.3$ & $98.4 \pm 2.8$ & $105.1 \pm 2.0$ & $0.17 \pm 0.01$ & $-30.8 \pm 2.2$ & $9.9 \pm 0.2$ & $98.1 \pm 2.3$ \\
\hline 30 & $110.3 \pm 3.1$ & $0.18 \pm 0.03$ & $-29.4 \pm 1.9$ & $9.8 \pm 0.5$ & $97.4 \pm 3.0$ & $107.5 \pm 1.8$ & $0.17 \pm 0.06$ & $-30.4 \pm 1.9$ & $9.9 \pm 0.2$ & $98.4 \pm 2.8$ \\
\hline 60 & $114.7 \pm 2.7$ & $0.19 \pm 0.04$ & $-28.1 \pm 3.1$ & $9.7 \pm 0.4$ & $97.1 \pm 1.9$ & $108.3 \pm 0.9$ & $0.18 \pm 0.03$ & $-30.1 \pm 1.4$ & $9.8 \pm 0.6$ & $97.9 \pm 1.7$ \\
\hline
\end{tabular}




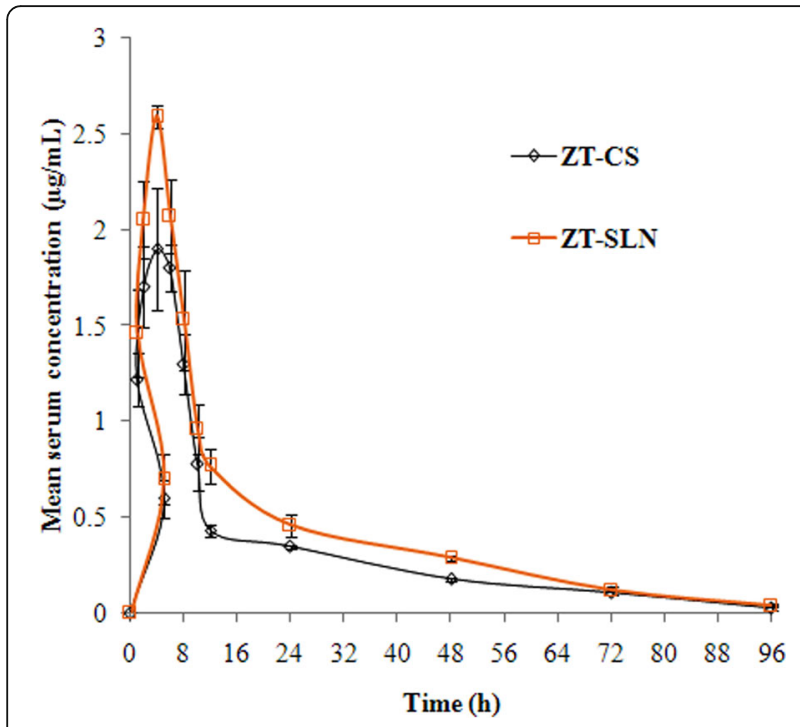

Fig. 6 Mean serum concentration - time profiles of zotepine from ZT-CS and optimized ZT-SLN formulation after oral administration in rats (mean $\pm S D, n=6$ )

stabilization as well. The presence of Poloxamer-188 in the development of SLNs could be favored for the maintenance of this ZP [31]. In general, ZP with $\pm 30 \mathrm{mV}$ could be considered as stable dispersion system. Formulations prepared with Dynasan ${ }^{\circ} 118$ and Dyanasn-114 showed above $-25 \mathrm{mV}$ and indicates stability. But, formulations F3 and F6 had below - $16 \mathrm{mV}$ of ZP.

The crystal lattice of the lipid matrix might be the reason for difference in the drug release from ZT-SLNs. Triglyceride formulations (Dyansan-118 and Dyna$\operatorname{san}^{\circ} 114$ ) showed slightly better release than mixed glyceride (Compritol ATO888) formulations. All the formulations showed burst release in initial $2 \mathrm{~h}$ and followed by slow prolonged release of the drug. This probably due to the unentrapped drug and the drug which present in the outer layer of the lipid showed burst effect. The drug embedded inside the lipid matrix slowly diffuse the drug as time progress.

Table 5 Pharmacokinetic parameters of zotepine from zotepine coarse suspension (ZT-CS) and zotepine solid lipid nanoparticle (ZT-SLN) formulations after oral administration in rats (mean \pm $\mathrm{SD}, n=6)$

\begin{tabular}{lll}
\hline Parameter & ZT-CS (F7) & ZT-SLN (F1) \\
\hline $\boldsymbol{C}_{\max }(\boldsymbol{\mu g} / \mathbf{m L})$ & $1.8 \pm 0.2$ & $2.4 \pm 0.6^{*}$ \\
$\boldsymbol{T}_{\max }(\mathbf{h})$ & 4 & 4 \\
$\mathbf{A U C} \boldsymbol{C}_{\text {total }}(\boldsymbol{\mu g} / \mathbf{m L}) . \mathbf{h}$ & $32.3 \pm 1.1$ & $43.3 \pm 2.2^{*}$ \\
$\boldsymbol{t}_{\mathbf{1 / 2}}(\mathbf{h})$ & $16.0 \pm 1.0$ & $18.3 \pm 2.3$ \\
MRT (h) & $19.7 \pm 2.6$ & $25.6 \pm 1.5^{*}$ \\
\hline
\end{tabular}

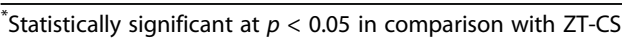

From the ex vivo results, a statistically significance difference $(p<0.05)$ was observed in percentage of permeation of ZT from SLN formulation compared with control formulation through rat intestine. The prolonged release of the ZT from the ZT-SLN formulations was observed from the in vitro release and ex vivo permeation studies.

The crystalline nature, surface morphology, and conversion of lipid nanoparticles into solid dosage forms by incorporating in capsules or compression to tablets were easy by the conversion of dispersion to solid form of the nanoparticles. This is generally achieved with either spray drying or freeze drying techniques. From the results of reconstituted lyophilized powder, about two to three-fold increase in the particle size and PDI of the formulation was observed after lyophilization. This could be due to the aggregation of the particles during the process of freeze-drying. But, there were no significant changes noticed in the other parameters. There were no significant changes assay, and EE in the reconstituted lyophilized SLN formulation indicates that there were no structural changes in the nanoparticle formulation.

From the DSC studies, in physical mixture, the intensity of drug peak was reduced compared with pure drug. It is known that the quantity of material used, especially in drug-excipient mixtures, could influence the peak shape and enthalpy. Thus, these minor changes in the melting endotherm of drug could be due to the mixing of drug and excipient, which lowered the purity of each component in the mixture, and this might not necessarily indicate potential incompatibility. Drug endothermic peak was absent in the lyophilized SLN formulation. The absence of endotherm peak of drug in lyophilized ZTSLN formulation unravels the conversion of native crystalline state of the drug to amorphous state. XRD studies also confirm the conversion of crystalline nature of ZT to amorphous form. This reduction in crystallinity or conversion to amorphous state of ZT from ZT-SLN was noticed in DSC analysis as well [32]. Physical stability of the formulation at refrigerated and room temperature was observed to be stable over 60 days.

Surface morphology study was conducted for pure drug and lyophilized ZT-SLN formulation. SEM picture revealed that pure drugs of $\mathrm{ZT}$ particles are possessed different shaped (cubic and spherical) but highly aggregated (Fig. 5a). Optimized formulations of ZT-SLN (F1) particles are nearly spherical shaped and also aggregated [33].

The PK studies in Wistar male rats confirmed the enhanced oral bioavailability of the ZT from ZT-SLN compared with coarse suspension formulation, after a single oral dose administration. From the $\mathrm{AUC}_{\text {total }}$, about 1.3fold improvement in the bioavailability observed from F1 formulation in comparison with F7 formulation. Similar type of results for improved oral delivery with 
SLN system was reported earlier [34-37]. Recently, ZT loaded NLCs also showed enhanced oral bioavailability in Wistar rats [38]. ZT-NLCs showed 1.6-fold enhancement in the oral bioavailability compared with coarse suspension. The NLCs are second generation of SLNs and exhibit better entrapment than SLNs. The expulsion of the drug is also avoided through NLCs. This could be the reason for changes (but non-significant) in the oral bioavailability in SLNs and NLCs. The improved oral bioavailability of SLNs of ZT could be due to the contribution of individual and/or combined effects of nanosized particles of the SLNs. The effective surface would influence the adhesion to GI tract. Consequently, there is increased contact time of the SLN particles. In addition, the soylecithin and poloxamer could alter the permeability characters of the GI membrane. The fatty acid chains present in the lipids of SLNs improve the uptake by lymphatic transport. This lymphatic transport minimizes the first-pass effect of the drug [39, 40].

\section{Conclusion}

ZT loaded solid lipid nanoparticles were successfully developed for the improved oral delivery. The ZT-SLN formulation was prepared with homogenization followed by sonication method. The optimized formulation was selected. DSC and XRD studies revealed the conversion to amorphous form of ZT in SLN formulation. In vitro and ex vivo permeation studies confirm the sustained release of the ZT from SLN formulation comparison with ZT coarse suspension as control formulation. Optimized ZT-SLN formulation was stable over 2 months at refrigerated and room temperature. Pharmacokinetic study in male Wistar rats revealed that there was 1.3 -fold improvement in oral bioavailability from SLN formulation when compared to coarse suspension. The overall results indicated that the lipid-based delivery systems considerably improved the bioavailability of ZT.

\section{Abbreviations \\ BCS: Biopharmaceutical classification system; CS: Coarse suspension; DSC: Differential scanning calorimetry; EE: Entrapment efficiency; GIT: Gastrointestinal tract; IS: Internal standard; NLCs: Nanostructured lipid carriers; PDI: Poly dispersity index; PK: Pharmacokinetics; SEM: Scanning electron microscope; SLNs: Solid lipid nanoparticles; XRD: X-ray diffractometer; ZP: Zeta potential; ZT: Zotepine}

\section{Acknowledgements}

The authors are thankful to M/s Symed labs, Hyderabad, for providing zotepine as a gift sample.

\section{Authors' contributions}

We declare that this work was done by the authors named in this article: ND conceived and designed and drafted the manuscript study. TC and DS carried out the laboratory work, collected and analyzed the data, and drafted the manuscript. NB supervised the work and drafted the manuscript. All authors have read and approved the final manuscript.

\section{Funding}

No funding was received.

\section{Availability of data and materials}

All necessary data generated or analyzed during this study are included in this published article. Any additional data could be available from the corresponding author upon request.

Ethics approval and consent to participate

The studies were conducted with prior approval of Institutional Animal Ethical Committee (IAEC/01/UCPSC /KU/2018).

\section{Consent for publication}

Not applicable

\section{Competing interests}

The authors declare that they have no competing interests.

Received: 4 March 2020 Accepted: 30 June 2020

Published online: 23 July 2020

\section{References}

1. Louis L, Harvey W (2017) Predicting oral drug absorption: mini review on physiologically- based pharmacokinetic models. Pharmaceutics 9(4):41. https://doi.org/10.3390/pharmaceutics9040041

2. Narendar D, Kishan V (2017) Candesartan cilexetil nanoparticles for improved oral bioavailability. Ther Del 8(2):79-88

3. Zhang L, Wang S, Zhang M, Sun J (2013) Nanocarriers for oral drug delivery. J Drug Target 21:515-527

4. Arun B, Narendar D (2015) Enhancement of solubility and dissolution rate of trandolapril sustained release matrix tablets by liquisolid compact approach. Asian J Pharma 9(4):290-297

5. Mei L, Haonan X, Jingzheng J et al (2017) Liquisolid technique and its applications in pharmaceutics. Asian J Pharm Sci 12:115-123

6. Nagaraj K, Narendar D, Kishan V (2017) Development of olmesartan medoxomil optimized nanosuspension using Box-Behnken design to improve oral bioavailability. Drug Dev Ind Pharm 43(7):1186-1196

7. Yanbin H, Wei-Guo D (2014) Fundamental aspects of solid dispersion technology for poorly soluble drugs. Acta Pharm Sin B 4(1):18-25

8. Palem CR, Narendar D, Satyanarayana G, Varsha BP (2016) Development and optimization of atorvastatin calcium-cyclodextrin inclusion complexed oral disintegrating tablets for enhancement of solubility, dissolution, pharmacokinetic and pharmacodynamic activity by central composite design. Int J Pharm Sci Nanotech 9(2):3170-3181

9. Serajuddin AT (2007) Salt formation to improve drug solubility. Adv Drug Deliv Rev 59(7):603-616

10. William NC, Christopher JHP (1996) Lipophilic prodrugs designed for intestinal lymphatic transport. Adv Drug Deliv Rev 19:149-169

11. Natalie LT, William NC, Christopher JHP (2008) Lipid- bsased delivery systems and intestinal lymphatic drug transport: a mechanistic update. Adv Drug Deliv Rev 60:702-716

12. Müller RH, Mäder K, Gohla S (2000) Solid lipid nanoparticles (SLN) for controlled drug delivery - a review of the state of the art. Eur J Pharm Biopharm 50(1):161-177

13. Mehnert W, Mäder K (2012) Solid lipid nanoparticles. production, characterization and applications. Adv Drug Deliv Rev 64:83-101

14. Wenyu W, Xiuli Z, Haiyang H, Dawei C et al (2010) Galactosylated solid lipid nanoparticles with cucurbitacin B improves the liver targetability. Drug Deliv 17(3):114-122

15. Tatke A, Dudhipala N, Janga KY et al (2019) In situ gel of triamcinolone acetonide-loaded solid lipid nanoparticles for improved topical ocular delivery: tear kinetics and ocular disposition studies. Nanomaterials 9(1):33. https://doi.org/10.3390/nano9010033

16. Ben G (2009) Zotepine: a clinical review. Expert Opin Drug Metab Toxicol 5(2):81-86

17. Tanaka O, Kondo T, Otani K, Yasui N, Tokinaga N, Kaneko S (1998) Single oral dose kinetics of zotepine and its relationship to prolactin response and side effects. Ther Drug Moni 20(1):117-119

18. Tanaka O (1996) Pharmacokinetics of zotepine and various factors affecting that of zotepine. Nihon Shinkei Seishin Yakurigaku Zasshi 16(2):49-52

19. Pailla SR, Tallur S, Rangaraj N, Ramavath R et al (2019) Intranasal zotepine nanosuspension: intended for improved brain distribution in rats. Daru 27 541-556 
20. Dalvadi H, Patel N, Parmar K (2017) Systematic development of design of experiments (DoE) optimised self-microemulsifying drug delivery system of zotepine. J Microencapsul 34:308-318

21. Mangesh B, Prashant U, Ashwini M (2017) Formulation and characterization of solid lipid nanoparticles for an anti-retroviral drug darunavir. App Nanosci 7(1-2):47-57

22. Dudhipala N, Veerabrahma K (2017) Improved anti-hyperlipidemic activity of rosuvastatin calcium via lipid nanoparticles: pharmacokinetic and pharmacodynamic evaluation. Eur J Pharm Biopharm 110:47-57

23. Narendar D, Kishan V (2014) Candesartan cilexetil loaded solid lipid nanoparticles for oral delivery: characterization, pharmacokinetic and pharmacodynamic evaluation. Drug Deliv 23:395-404

24. Narendar D, Kishan V (2015) Pharmacokinetic and pharmacodynamic studies of nisoldipine-loaded solid lipid nanoparticles developed by central composite design. Drug Dev Ind Pharm 41(12):1968-1977

25. Arun B, Narendar D, Kishan V (2018) Development of olmesartan medoxomil lipid-based nanoparticles and nanosuspension: preparation, characterization and comparative pharmacokinetic evaluation. Artif cells Nanomed Biotechnol 46(1):126-137

26. Mai MG, Sana MM, Marwa AS (2017) Hexagonal liquid crystalline nanodispersions proven superiority for enhanced oral delivery of rosuvastatin: in vitro characterization and in vivo pharmacokinetic study. J Pharma Sci 106:3103-3112

27. Mohammad M, Hadi V, Parvin ZM (2018) Bortezomib-loaded solid lipid nanoparticles: preparation, characterization, and intestinal permeability investigation. Drug Dev Ind Pharm 44(10):1598-1605

28. Thirupathi G, Swetha E, Narendar D (2017) Role of isradipine loaded solid lipid nanoparticles in the pharmacodynamic effect of isradipine in rats. Drug res 67(03):163-169

29. Narendar D, Karthik YJ (2017) Lipid nanoparticles of zaleplon for improved oral delivery by Box-Behnken design: optimization, in vitro and in vivo evaluation. Drug Dev Ind Pharm 43(7):1205-1214

30. Narendar D, Yadav JK, Thirupathi G (2018) Comparative study of nisoldipineloaded nanostructured lipid carriers and solid lipid nanoparticles for oral delivery: preparation, characterization, permeation and pharmacokinetic evaluation. Artif cells Nanomed Biotechnol 46:616-625

31. Suvarna D, Narendar D, Kishan V (2015) Preparation, characterization and in vivo evaluation of rosuvastatin calcium loaded solid lipid nanoparticles. Int J Pharm Sci Nanotech 8(1):2779-2785

32. Xin WL, Xiao HL, Li QZ, Hong ZM (2008) Preparation, characterization, and in vitro release of chloramphenicol loaded solid lipid nanoparticles. J Dispe Sci Techno 29(9):1214-1221

33. Pitta SK, Dudhipala N, Narala A, Veerabrahma K (2018) Development and evaluation of zolmitriptan transfersomes by Box-Behnken design for improved bioavailability by nasal delivery. Drug Dev Ind Pharm 44(3): 484-492

34. Dudhipala N, Thirupathi G (2020) Neuroprotective effect of ropinirole loaded lipid nanoparticles hydrogel for Parkinson's disease: preparation, in vitro, ex vivo, pharmacokinetic and pharmacodynamic evaluation. Pharmaceutics 12(5):448

35. Reddy ND, Reddy GP (2018) Capecitabine lipid nanoparticles for anti-colon cancer activity in 1, 2-dimethylhydrazine induced colon cancer: preparation, cytotoxic, pharmacokinetic and pathological evaluation. Drug dev Ind pharm 44(10):1572-1582

36. Sandeep V, Narendar D (2016) Arjun N and Kishan V (2016) Lacidipine loaded solid lipid nanoparticles for oral delivery: preparation, characterization and In vivo evaluation. IJPSN 9(6):3524-3530

37. Gondrala UK, Dudhipala N, Kishan V (2015) Preparation, characterization and in vivo evaluation of felodipine solid lipid nanoparticles to improve the oral bioavailability. Int J Pharm Sci Nanotech 8(4):2995-3002

38. Tirumalesh C, Suram D, Dudhipala N, Banala N (2020) Enhanced pharmacokinetic activity of zotepine via nanostructured lipid carrier system in wistar rats for oral application. Pharm Nanotechnol 8(2):148-160

39. Arik D, Amnan $\mathrm{H}$ (2007) The effect of different lipid based formulations on the oral absorption of lipophilic drugs: the ability of in vitro lipolysis and consecutive ex vivo intestinal permeability data to predict in vivo bioavailability in rats. Eur J Pharm Biopharm 67:96-105

40. Narendar D (2019) A comprehensive review on solid lipid nanoparticles as delivery vehicle for enhanced pharmacokinetic and pharmacodynamic activity of poorly soluble drugs. Int J Pharm Sci Nanotech 12(2):4421-4440

\section{Publisher's Note}

Springer Nature remains neutral with regard to jurisdictional claims in published maps and institutional affiliations.

\section{Submit your manuscript to a SpringerOpen ${ }^{\circ}$ journal and benefit from:}

- Convenient online submission

- Rigorous peer review

- Open access: articles freely available online

- High visibility within the field

- Retaining the copyright to your article

Submit your next manuscript at $\boldsymbol{\nabla}$ springeropen.com 